\title{
REQUERIMIENTOS ACADÉMICOS EN ESTUDIANTES UNIVERSITARIOS: EL CAMINO RECORRIDO POR EL CENTRO DE APOYO AL RENDIMIENTO ACADÉMICO Y DE EXPLORACIÓN VOCACIONAL DE LA UC
}

\author{
Ana María Hojas, María José Anais, Angélica Bustos, \\ Cecilia Letelier, Soledad Zuzulich ${ }^{1}$
}

RESUMEN

El presente artículo se centra en describir la experiencia del Centro de Apoyo al Rendimiento Académico y de Exploración Vocacional de la Pontificia Universidad Católica de Chile. Específicamente describe la evolución de sus servicios desde su etapa de diseño y cómo, luego de ocho años de funcionamiento, estos se han desarrollado y diversificado para responder a las necesidades académicas de los alumnos de esta universidad.

El recorrido realizado por el centro pretende dar cuenta de la relevancia de abordar las temáticas asociadas al rendimiento académico y el estudio como un área que requiere un equipo especializado de profesionales.

Palabras clave: rendimiento académico, habilidades de estudio, estudiantes universitarios, apoyo académico

\section{ACADEMIC REQUIREMENTS IN UNIVERSITY STUDENTS: THE EXPERIENCE OF THE CHILEAN CATHOLIC UNIVERSITY'S CENTER FOR SUPPORTING ACADEMIC PERFORMANCE AND VOCATIONAL EXPLORATION}

ABSTRACT

This article describes experiences from the Centre for Supporting Academic Performance and Vocational Exploration of the Pontifical Catholic University of Chile. In particular, it describes the evolution of its services and how these services have developed and diversified, after eight years operating, according to academic needs of students in this University.

The experience of the Centre shows the importance of addressing issues associated with academic performance and study as areas that require specialized professionals.

Keywords: academic achievement, study skills, college students, academic support

1 Centro de Apoyo al Rendimiento Académico y de Exploración Vocacional, Dirección de Asuntos Estudiantiles, Pontificia Universidad Católica de Chile. Santiago. Chile. Contacto: ahojas@uc.cl 


\section{REQUERIMIENTOS ACADÉMICOS EN ESTUDIANTES UNIVERSITARIOS: EL CAMINO RECORRIDO POR EL CENTRO DE APOYO AL RENDIMIENTO ACADÉMICO Y DE EXPLORACIÓN VOCACIONAL DE LA UC}

La transición desde el colegio a la universidad implica importantes desafíos para los alumnos, tanto en el plano académico como personal. Las exigencias son diferentes, lo que requiere desarrollar estrategias de estudio, nuevas fuentes de motivación y mayor autorregulación en su proceso de aprendizaje, entre otros factores (Pascarella y Terenzini, 1991; García y Ramírez, 2005). En este contexto nace, en la Unidad de Apoyo Psicológico, el Centro de Apoyo al Rendimiento Académico y de Exploración Vocacional (en adelante CARA UC), de la Pontificia Universidad Católica (UC), teniendo como propósito fundamental apoyar el desempeño y aprendizaje de los alumnos, en tanto factor protector de su salud mental (Susperreguy, Flores, Micin y Zuzulich, 2007).

La misión del Centro es ofrecer, desde el marco de la psicología educacional, alternativas que permitan potenciar el desarrollo de habilidades académicas, para que los jóvenes vivan con éxito y satisfacción su proceso de aprendizaje, estudio, y la etapa universitaria en general. Los objetivos del centro se orientan a pesquisar factores de riesgo ligados al proceso de estudio, desarrollando y promoviendo un aprendizaje estratégico y autorregulado, junto con apoyar la búsqueda de la definición vocacional, según los intereses y preferencias profesionales de los estudiantes.

\section{Los inicios del CARA UC}

El CARA UC surge en el contexto de la Unidad de Apoyo Psicológico (UAP), dependiente de la Dirección de Salud Estudiantil, debido al crecimiento de consultas relacionadas con el rendimiento y la vocación. En el año 2002 se definió el desarrollo de un apoyo con 
herramientas específicas ligadas al aprendizaje. Fue así como surgió el taller "Mejorando mi Rendimiento Académico", cuya demanda aumentó rápida y progresivamente, manifestándose la necesidad de contar con profesionales especializados.

Sumado a lo anterior, y asumiendo que la universidad es responsable de proveer las condiciones para que sus estudiantes aprendan y rindan adecuadamente, el año 2004 se estableció un equipo de profesionales para apoyar a los estudiantes de la UC. Desde el comienzo se señaló como un soporte transversal dispuesto para todos, tanto a quienes presentaran problemas de rendimiento como para quienes buscaran potenciar su proceso de estudio o exploración vocacional.

Pero ¿cómo podía explicarse la existencia de un centro de apoyo al rendimiento académico en una de las universidades con los puntajes de ingreso más altos del país?; ¿por qué necesitarían algún tipo de ayuda?

Desde sus comienzos, las inquietudes y requerimientos de los estudiantes que llegaban hasta el centro eran coherentes con lo que la literatura especializada resalta: la importancia de las estrategias y habilidades apropiadas para los requerimientos del estudio universitario, aspecto que no necesariamente se ha desarrollado en el colegio. Estas habilidades serían de tal relevancia, que se ha sugerido que las instituciones educativas las enseñen (Ellis, 2005).

Esa necesidad se refleja también en la tasa de deserción en primer año, que en las instituciones del Consejo de Rectores de las Universidades Chilenas (CRUCH) es del 19\%, y que tiene entre sus causas más determinantes los problemas vocacionales y de rendimiento (Universidad de Chile, 2008). En la UC, entre los años 2005 y 2010, la tasa de retención se ha mantenido entre 88\% y 93,4\% (Pontificia Universidad Católica de Chile [UC], 2011), que aun siendo mayor que las de otras instituciones, también invita a abordar posibles necesidades académicas de sus estudiantes.

La existencia del CARA UC, focalizado en apoyar las necesidades asociadas al rendimiento académico o al ámbito vocacional de 
los alumnos, ha normalizado y validado este tipo de inquietudes, incorporándose a las diversas alternativas de ayuda que ofrece la universidad, siendo ampliamente conocido por los alumnos (en una encuesta dirigida a los estudiantes de pregrado ${ }^{2}$, el 82,3\% de quienes respondieron indicaban conocer el CARA UC).

Paulatinamente, al abordar estas temáticas, el equipo de profesionales del centro ha mostrado el aporte de la psicología educacional en este ámbito, con modalidades de trabajo distintas (y en ocasiones complementarias) al abordaje clínico. Se ha evidenciado además que estos servicios tienen un foco específico: el proceso de estudio y/o exploración vocacional como un factor protector de salud mental.

La demanda por los servicios ofrecidos (sesiones diagnósticas, talleres, tutorías, atención psicoeducativa y el curso) ha aumentado. El año 2004, 310 estudiantes los utilizaron. El año 2011, 2.977 estudiantes tomaron alguno de sus servicios. Así, en sus ocho años de funcionamiento, 16.259 estudiantes de la UC han participado de alguna de las intervenciones psicoeducativas de las que dispone el centro, incluyendo el curso.

Este crecimiento en número de participantes y de los servicios ofrecidos, ha implicado un aumento sostenido en el equipo de profesionales, compuesto actualmente por cuatro psicólogas con formación en psicología educacional, y un equipo de alrededor de diez profesores que se incorporan semestralmente para dictar los cursos (servicio que se explicará más adelante). La demanda sostenida ha sugerido la necesidad de una nueva infraestructura; lo que implicó contar con nuevas dependencias el año 2006, y con un cambio proyectado para el 2013 (ver gráfico $n^{\circ} 1$ ).

Asimismo, la gran cantidad de inscripciones en los servicios hizo necesario contar con un sistema automatizado en línea, para el manejo de la base de datos y optimización de los recursos, permitiendo procesar las solicitudes y hacer seguimiento de los participantes.

2 Encuesta enviada a todos los alumnos de pregrado de la PUC en octubre de 2009 (CARA UC, 2009). 
Gráfico n. ${ }^{\circ}$ 1: Participantes por servicio 2004 a 2011

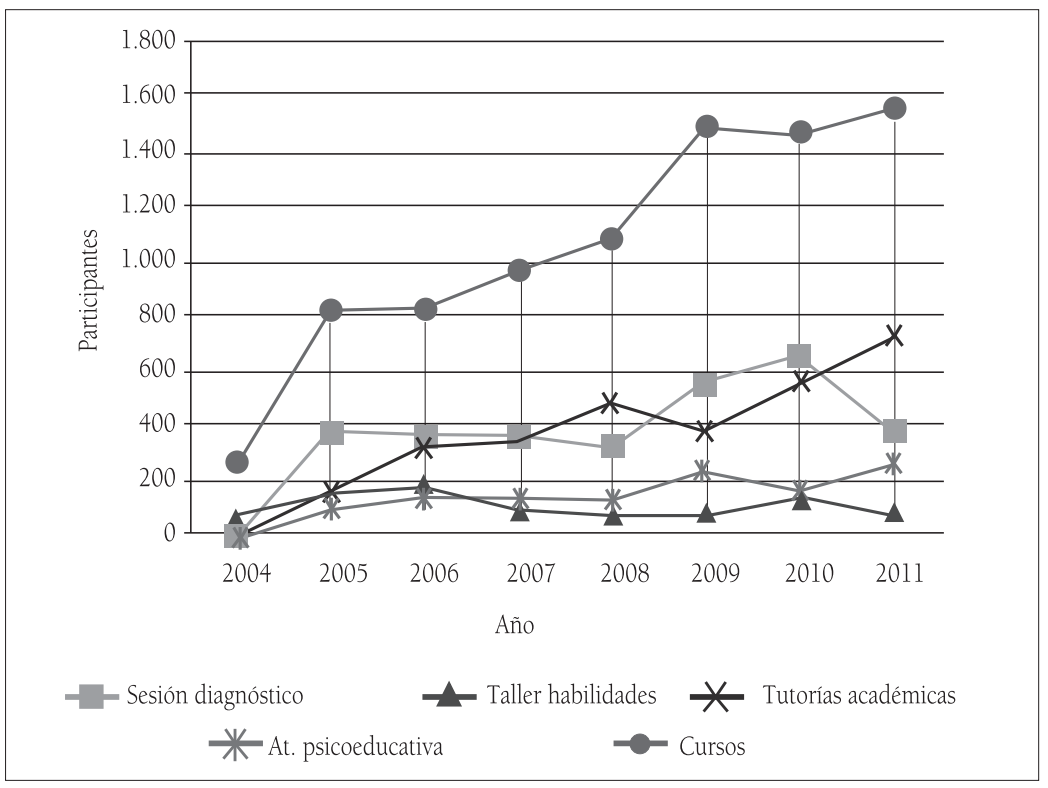

\section{Servicios que ofrece el CARA}

Se seleccionaron cuatro servicios de los que ofrece el centro; el curso, tutorías, atención psicoeducativa y talleres, por ser los de mayor inscripción, porque han presentado cambios interesantes de reportar, y permiten mostrar la diversidad de modalidades con las que es posible trabajar las temáticas asociadas al rendimiento académico y la exploración vocacional, en el contexto universitario. En cada uno, junto con la descripción del servicio, se agregan datos estadísticos cuando corresponda.

\section{a. Curso de hábitos y estrategias de estudio}

Este curso busca fomentar el reconocimiento y comprensión de las variables que influyen en el rendimiento académico. Se trabaja en una modalidad de curso-taller, abordando elementos teóricos de aplicación y reflexión personal. Se imparte como parte de la malla curricular en algunas facultades y como optativo de formación general. 
Debido a sus características, tiene un cupo de 22 estudiantes por sección. Implica una carga académica de 5 créditos $^{3}$ y para aprobarlo se requiere obtener un promedio sobre 4,0, y cumplir con $80 \%$ de asistencia a clases, de modo de asegurar la reflexión, participación y ejercitación suficiente. La demanda de los cursos se ha quintuplicado: el 2004 se comenzó con 13 secciones anuales, llegando a 74 en el 2011.

La modalidad teórico-práctica permite comprender en profundidad que el proceso de estudio no es posible de explicar por una sola variable ni por una sumatoria de elementos, sino que por variables que, relacionadas entre sí, finalmente afectan el aprendizaje y rendimiento (ver modelo figura 1). Comprender esta complejidad es relevante para intervenir en el proceso de estudio. Se espera que los alumnos incorporen los conceptos, llevándolos a su propio estudio, lo que constituye el principal aporte de este curso.

La evaluación de los alumnos es positiva. Entre el segundo semestre de 2010 y primer semestre de 2011 por ejemplo, el 91,2\% de los alumnos "recomendaría el curso a un amigo". Entre sus principales aprendizajes refieren la organización del tiempo, estrategias de estudio y el conocimiento y optimización de la concentración, apuntando a una comprensión activa del aprendizaje (Bustos y Letelier, 2011).

En esa misma línea, las escuelas de ingeniería y de enfermería sistemáticamente solicitan el curso para sus estudiantes de primer año. Así, por ejemplo, 3.214 estudiantes de ingeniería han pasado por el curso y muchos de ellos continúan usando sus servicios; tutorías o atención psicoeducativa.

\section{b. Tutorías académicas de pares}

Hasta el año 2005, las alternativas de atención del CARA UC estaban enfocadas en el proceso de estudio propiamente tal, entonces surge

3 En la Universidad Católica los alumnos toman cada semestre alrededor de 50 créditos, lo que equivale -con cierta variabilidad-a aproximadamente 5 cursos. 
la inquietud de entregar un apoyo específico en asignaturas de alta repitencia.

Las tutorías académicas consisten en sesiones grupales de seis alumnos, a cargo de un tutor que los acompaña aclarando dudas y resolviendo en conjunto ejercicios sobre contenidos de un ramo en particular, enfocándose en el desarrollo de habilidades específicas a los contenidos de ese curso. Se realizan una vez a la semana, durante cuatro semanas, pudiendo renovar su inscripción. Coherente con el abordaje del centro, el foco principal del servicio es la promoción del aprendizaje estratégico y el estudio activo.

El tutor es un alumno de la universidad que ha cursado el ramo del cual realiza tutoría y que ha sido seleccionado por el equipo CARA UC sobre la base de sus habilidades pedagógicas, responsabilidad y motivación por enseñar. Asume un rol de facilitador del aprendizaje, modelando formas de resolución y estudio de contenidos, en un clima de confianza, que es valorado por los estudiantes: "[el tutor] sabía mucho y se preocupaba de que aprendieras". O reconocen que "... sirve para cuestionarse la materia y buscar entre todos la solución, así el conocimiento se hace más propio" (CARA UC, 2011).

En el proceso se generan vínculos importantes: los tutores se comprometen con los alumnos realizando un seguimiento de sus aprendizajes y requerimientos. Los participantes por su parte, también se comprometen, generándose grupos de estudio.

Los tutores reciben capacitación y acreditación de sus competencias. Su continuidad como tutor depende de su autoevaluación, de la evaluación de los profesionales del centro y la de los alumnos participantes. A partir de este proceso de formación y evaluación se ha ido conformando un equipo de estudiantes con experiencia y capacidad de aportar a sus pares, lo que permite asegurar que las tutorías constituyen un espacio de aprendizaje para los estudiantes, y de formación para los tutores.

Las tutorías se han consolidado como un servicio de apoyo relevante; hasta el 2011, 2.940 estudiantes han asistido a tutorías de 
diferentes cursos, mayoritariamente estudiantes de $1^{\text {er }}$ año, quienes por lo general corresponden a la mayoría de los participantes (entre el 2009 y 2011 se distribuyó entre el 70\% a 87\% del total).

\section{c. Atención psicoeducativa}

Esta modalidad surgió debido a la presencia de inquietudes específicas de estudio por parte de asistentes a los cursos y talleres ofrecidos, los que requerían de un espacio de trabajo individual, con supervisión y seguimiento.

Debido a que era un nuevo servicio y que existían pocos antecedentes en Chile en este tipo de intervención, la experiencia acumulada de los profesionales del CARA UC llevó a desarrollar un modelo de trabajo que ha sido especialmente importante en el perfeccionamiento de este servicio (ver figura n. ${ }^{\circ} 1$ ).

Actualmente existen tres modalidades de trabajo: Modalidad de habilidades de aprendizaje estratégico, centrada en el desarrollo de hábitos de estudio; habilidades de concentración y memorización, estrategias de gestión de tiempo, entre otros. (2) Modalidad motivación académica, que aborda este aspecto relacionándolo con creencias respecto del aprendizaje, metas académicas y autorregulación, y (3) Modalidad de exploración vocacional que está dirigida a alumnos con inquietudes vocacionales, que no se proyectan en su carrera o no tienen claridad sobre alternativas para explorar.

En general, los estudiantes relatan que para obtener buenos resultados académicos en la enseñanza media se requería de poco esfuerzo, por lo que es probable pesquisar en el contexto universitario estudiantes con baja sistematicidad e inadecuados niveles de autorregulación, siendo esta una situación independiente del tipo de colegio de proveniencia y de la carrera en curso. Esta condición se hace crítica al ingreso a la universidad, requiriendo apoyo específico en este ámbito.

Por otra parte, la literatura describe que las personas con múltiples habilidades y/o intereses, pueden enfrentar dificultades 
o desafíos al escoger una carrera entre tantas opciones (Colangelo y Assouline, 2000) precisamente por su "multipotencialidad" y las expectativas de sus profesores, sus familias, y de sí mismos.

Así, en la modalidad vocacional se observa que les cuesta priorizar sus intereses. O que la influencia de la familia y de sus propias autoexigencias puede generar dificultades en la elección profesional. En la intervención psicoeducativa, se aborda explicitando y respetando la cultura familiar, pero también enfatizando la importancia de un proyecto de vida y metas a largo plazo. De esta forma, se fomenta la profundización en el autoconocimiento, con apoyo de un instrumento especialmente creado con esta finalidad, el Cuestionario de intereses vocacionales (CARA UC y Mide UC, 2009).

Desde sus inicios, 1.130 estudiantes han asistido a atención psicoeducativa. El año 2011, el 84,7\% asistió a entre una y cuatro sesiones. En ese mismo periodo, el 43,5\% de quienes tomaron una hora buscaba trabajar en estrategias de estudio, el 33,2\% en exploración vocacional y el 4,9\% en motivación académica (CARA UC, 2011).

\section{d. Talleres de habilidades académicas}

El taller "Mejorando mi rendimiento académico" fue uno de los primeros servicios del centro y abordaba las principales variables que influyen en el rendimiento académico. Sin embargo, en la medida que se fueron incorporando otros servicios, aparecieron temas más específicos, para tratar en esta modalidad de intervención. Actualmente se ofrecen los siguientes talleres:

- "Estudiar para rendir mejor", que es una reformulación del taller "Mejorando mi rendimiento académico", pero centrado en el proceso de estudio propiamente tal.

- "Usando el tiempo a mi favor", centrado en el uso y gestión del tiempo en el contexto y condiciones de vida de estudiantes universitarios.

- "Entrenando la concentración y memoria", centrado en aprender el funcionamiento de procesos de atención, concentración y memoria, para ejercitar y optimizar dichas habilidades. 
- "Entrenamiento en evaluaciones y presentaciones orales", focalizado en elementos propios del diseño de una presentación y ejercitación de habilidades de expresión oral, abordando las evaluaciones de este tipo.

Todos los talleres constituyen una instancia acotada de intervención (de dos a cuatro sesiones, dependiendo del taller), donde se conocen las habilidades para desarrollar y se ponen en práctica en la sesión y en el proceso de estudio personal.

A diferencia de los otros servicios del centro, con el tiempo ha disminuido la demanda por talleres; el año 2006 participaron 170 estudiantes, el 2010 fueron 138 y el 2011, 88. Esto probablemente se deba a la diversificación de servicios, a ciertas dificultades con incompatibilidad de horarios para participar y en especial a la existencia del curso y las temáticas que este abarca. Esto ha implicado ajustes en la oferta de talleres por periodo, en el número de sesiones y una permanente revisión de este servicio. Sin embargo se ha mantenido, considerando la evaluación positiva de quienes participan. Así, el 100\% de quienes asistieron al taller completo en 2010 y 2011 lo recomendaría, aludiendo a aspectos como "porque me sirvió mucho, y como a mí, sé que a otros alumnos les mejoraría su forma de estudio" (CARA UC, 2011).

\section{Enfoque del CARA UC para responder a las necesidades de los alumnos de la UC}

En los distintos servicios del centro así como en sus políticas y funcionamiento general, hay elementos transversales presentes desde sus inicios que se han ido nutriendo de la experiencia. Un elemento esencial se refiere a que los participantes del CARA UC son agentes activos y constructivos en su aprendizaje, pudiendo supervisarlo y regularlo en función de ciertas metas (González, Valle, Rodríguez y Piñeiro, 2002). Esto se expresa en las modalidades de trabajo y también en aspectos concretos como la asistencia y puntualidad en los servicios.

El quehacer del centro se sustenta en una comprensión del rendimiento académico como una variable dependiente de 
la interacción de distintas variables relacionadas entre sí. Para sistematizarlo se hizo necesario generar un modelo propio que fuera coherente con los antecedentes reportados por la literatura, pero especialmente con la experiencia de trabajo de este.

Como se observa en la figura 1 , en el rendimiento académico interactúan los procesos cognitivos, afectivos, la gestión de recursos del alumno y el proceso de estudio mismo, en sus distintas fases y niveles. En este contexto, cobra especial importancia la motivación académica que sustenta el estudio en sus distintas variables y momentos. Este modelo se centra en aquellas que el mismo alumno puede intervenir, reflejando lo más propio del centro. Se incluyen las características personales y el contexto académico que, aun estando fuera del control del alumno, son aspectos que debe considerar estratégicamente.

Los distintos servicios del centro se focalizan en ciertas variables. En atención psicoeducativa se pueden abordar todas las variables del proceso de estudio, considerando también las características personales o del contexto que pueden impactar en el aprendizaje. Las tutorías, por su parte, se focalizan en el proceso de estudio mismo, tanto de aspectos del contexto académico como conocimientos específicos. Finalmente, el curso aborda de manera teórica y práctica todos los elementos asociados al proceso de estudio. 
Figura 1: Modelo de rendimiento académico CARA UC

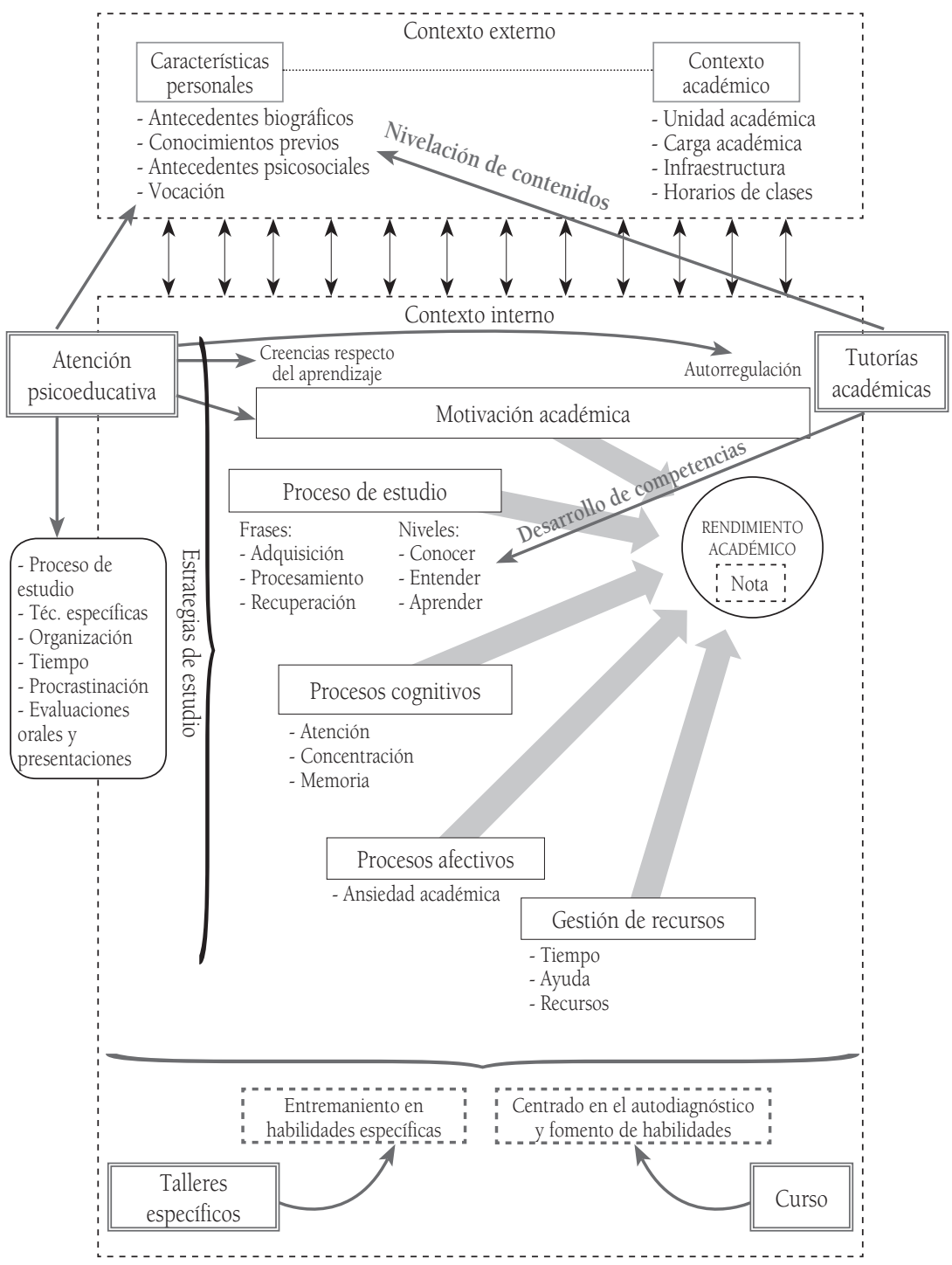

Este modelo ha sido de gran utilidad para transmitir a los mismos estudiantes aquellos aspectos que están en la base del rendimiento académico, y que no son fácilmente observables. Pero especialmente, invita a abordar el proceso de estudio de manera específica, acotada y 
multicausal, comenzando de manera paulatina a trabajar -por ejemploen alguna de las variables del proceso de estudio, asumiendo que, al existir interrelación, tendrá efectos en el proceso en general.

\section{Conclusiones y desafíos}

El presente artículo se ha centrado en el recorrido realizado por el CARA UC para apoyar a los estudiantes de esta universidad en el logro de un mejor aprendizaje, buen rendimiento y, sobre todo, de mejor calidad de vida.

El compromiso de la universidad para responder a las necesidades de sus estudiantes se ha traducido en un trabajo sistemático con ellos, lo que ha permitido aprender acerca de los procesos de aprendizaje y estudio e ir paulatinamente ajustando y creando una oferta de servicios según sus requerimientos y características. En la actualidad es un referente para los alumnos que buscan optimizar su aprendizaje y rendimiento o sentirse satisfechos con su vocación profesional.

Dicha experiencia ha permitido adaptar estos servicios para brindar un apoyo adecuado y desarrollar algunos específicos. Por ejemplo, para alumnos con dificultades importantes en su rendimiento (causal de eliminación por rendimiento académico, primera vez), mediante un Programa Remedial. También, se han desarrollado sesiones diagnósticas especiales para ciertas carreras y talleres a petición de facultades e incluso de agrupaciones estudiantiles.

La labor de estos años ha permitido además situar la temática del rendimiento académico y el desarrollo de habilidades para el estudio en contextos universitarios, como un ámbito que requiere de un abordaje especializado, en este caso, desde la psicología educacional. Estos logros invitan a continuar revisando la labor del centro, y a plantear nuevas metas y desafíos.

El primero se refiere a continuar y mejorar la expansión, difusión interna y focalización de la oferta de servicios del centro, aun cuando se ha ido posicionando cada vez más en la comunidad 
universitaria. En la misma línea, es importante profundizar más en conocer a los alumnos: sus modalidades de aprendizaje, creencias y necesidades asociadas, para asegurar la pertinencia de los servicios y metodologías de trabajo en función de estas particularidades.

Así, sin olvidar que el foco del centro está en ofrecer un servicio a los estudiantes, de manera incipiente se espera investigar en temas asociados al rendimiento académico y seguimiento de quienes utilizan los servicios. Es por esto que se está trabajando en el diseño de un instrumento que evalúe estrategias de estudio en este contexto, y que reemplace al Inventario de procesos de estudio UC (Rosas, Manzi y Moore, 1987) que actualmente se emplea.

Mediante el CARA UC, esta universidad ha sido pionera en asumir la integralidad de sus alumnos, comprendiendo la relevancia de acoger sus fortalezas y debilidades, reconociendo la heterogeneidad existente, la importancia del fomento del esfuerzo, la autonomía y la motivación por aprender. Así, se plantea la formación profesional resaltando la importancia de una experiencia de vida estudiantil saludable, que permita expresar al máximo las potencialidades de aprendizaje de cada uno de sus alumnos. Y en ese sentido, aportar a la cultura de la universidad y a la sociedad.

Agradecemos la colaboración del equipo del CARA UC en la realización de este artículo y a su permanente labor en el centro.

\section{Referencias bibliográficas}

Bustos, A.; Letelier, C. (2011). Curso de hábitos y estrategias de estudio. Ponencia presentada en I Jornada Nacional sobre Apoyo a Estudiantes Universitarios Iniciales, octubre de 2011, Valdivia, Chile.

CARA UC y MIDE UC (2009). Cuestionario de Intereses Vocacionales. Instrumento no publicado: Santiago, Chile.

Colangelo, N.; Assouline, S. (2000). Counseling gifted students. En K. Heller, F. Mönks, R. Sternberg, R. Subotnik (eds.), International Handbook of Giftedness and Talent (pp. 595-607). Oxford: Pergamon.

Ellis, J. (2005). Metacognición, aprendizaje autorregulado y estrategias de estudio. En Aprendizaje Humano (pp. 365-403). Madrid: Pearson Practice Hall. 
García, F.; Ramírez, P. (2005). Caracterización de las estrategias de aprendizaje en estudiantes en formación de Pedagogía en Educación General Básica. Boletín de Investigación Educacional, 20(2), pp. 189-210.

González, R.; Valle, A.; Rodríguez, S.; y Piñeiro, I. (2002). “Autorregulación del aprendizaje y estrategias de estudio”. En Pineda, J., Núñez, J. Álvarez, L. y Soler, E. (eds.), Estrategias de Aprendizaje. Concepto, evaluación e intervención (pp. 17-38). Madrid: Ediciones Pirámide.

Pascarella, E.; Terenzini, P. (1991). How college affects students. San Francisco: Jossey-Bass.

Pontificia Universidad Católica de Chile, CARA UC (2009). Informe de estadísticas de servicios. Documento no publicado: Santiago, Chile.

CARA UC (2009). Informe de aplicación cuestionario CARA. Documento no publicado. Pontificia Universidad Católica de Chile, Santiago, Chile.

CARA UC (2010). Informe de estadísticas de servicios. Documento no publicado. Pontificia Universidad Católica de Chile, Santiago, Chile.

CARA UC (2011). Informe de estadísticas de servicios. Documento no publicado. Pontificia Universidad Católica de Chile, Santiago, Chile.

Pontificia Universidad Católica de Chile (2011). Dirección de Análisis Institucional y Planificación. Estadísticas 2005 a 2010. Documento no publicado: Santiago, Chile.

Rosas, R.; Manzi, J.; Moore, C. (1987). Construcción y estandarización del inventario de procesos de estudio UC (IPEUC). Santiago de Chile: Escuela de Psicología, Universidad Católica de Chile.

Susperreguy, M.I.; Flores, A.; Micin, S.; Zuzulich, S. (2007). Apoyo académico en la educación superior: descripción de la experiencia y perfil de los alumnos que participan en el CARA UC. Revista Calidad en la Educación, n. ${ }^{\circ} 26$, pp. 307-333.

Universidad de Chile, Departamento de Economía, (2008). Estudio sobre causas de la deserción universitaria. Santiago: Centro de Microdatos.

Recibido: 30/03/2012

Aceptado: 19/04/2012 